\title{
Clinical analysis of moderate-to-deep-sedation by nonmedical sedation practitioners in 597 patients undergoing gastrointestinal endoscopy: a retrospec- tive study
}

Authors

Institution
Hermanus Vaessen, Elisabeth Bruens, Johannes Knape

Department of Anesthesiology, Intensive Care and Emergency Medicine, University Medical Centre Utrecht, the Netherlands submitted:

2. November 2015

accepted after revision:

8. February 2016

\section{Bibliography}

DOI http://dx.doi.org/

10.1055/s-0042-103238

Published online: 8.4.2016

Endoscopy International Open

2016; 04: E564-E571

(c) Georg Thieme Verlag KG

Stuttgart · New York

E-ISSN 2196-9736

\section{Corresponding author}

\section{H. H. B. (Paul) Vaessen, RNA,}

PSA Specialist

University Medical Centre Utrecht

Department of Anesthesiology, Intensive Care and Emergency

Medicine

Heidelberglaan 100

Huispostnr.: F02.811

3584 CX Utrecht

The Netherlands

Fax: + 31-30-7555442

H.H.B.Vaessen@umcutrecht.nl
Background and study aim: The purpose of this study was to evaluate whether moderate-todeep sedation with propofol and alfentanil can be administered safely by nonmedical sedation practitioners, and the outcomes of this practice in the Netherlands. We retrospectively analyzed the occurrence of sedation-related complications in patients undergoing gastrointestinal endoscopic procedures.

Patients and methods: In this study, 597 adult patients consecutively underwent upper gastrointestinal endoscopic procedures. The health status of the patients was screened according to a standardized protocol, and the patients were sedated by trained nonmedical sedation practitioners. Their vital signs were continuously monitored and recorded. All patients received oxygen, and the depth of sedation was continuously assessed and recorded. Mild and severe complications were recorded and analyzed.

\section{Introduction}

\section{$\nabla$}

Diagnostic and therapeutic gastrointestinal (GI) endoscopic procedures have become standard tools for the investigation and surveillance of GI disease. These often-complex procedures may be uncomfortable and painful for patients. Moderate-to-deep sedation is frequently used to relieve discomfort and improve working conditions for endoscopists [1].

Sedation is considered to be a risky medical procedure $[2,3]$. Traditionally, benzodiazepines, such as midazolam [4], have frequently been used for that purpose, often in combination with fentanyl [5] or meperidine [6]. However, many patients report bad experiences [7] and unpleasant memories of their procedures, refusing to undergo similar sedation during later endoscopic procedures. Sedation with propofol (2,6-diisopropylphenol) and a short-acting opioid is found to be comfortable by many patients, improves operational effi-
Results: All patients recovered uneventfully, and no mortality occurred. Overall, of the 597 sedated patients, 85 had mild and 4 had severe complications. Hypoxemia and upper airway obstruction, which were easily managed by trained nonmedical sedation practitioners, were the most common events. Hypotension was rare. No signs or symptoms suggestive of aspiration were reported. Conclusion: Moderate-to-deep sedation has been and continues to be a risky medical procedure. Serious complications of propofol/opioid-based sedation, especially respiratory and cardiovascular adverse events, may occur. These complications need to be recognized rapidly and appropriately managed. Our study shows that well-trained nonmedical sedation practitioners can be entrusted to take responsibility for the safe administration of moderate-to-deep sedation.

ciency, results in a short recovery time [8], and is more easily controlled than sedation with midazolam-based regimens [9].

The shortage of medical practitioners who can undertake the direct, personal, specialist-based supervision of moderate-to-deep sedation in a significant number of patients in the Netherlands [10] has led to initiatives to transfer responsibilities for such sedation to health care personnel other than anesthesiologists. Therefore, a pilot program for sedation skills training was developed in the departments of anesthesiology and gastroenterology at the University Medical Centre Utrecht (UMCU) and the Amsterdam Medical Centre (AMC) as part of an effort to shift or reshuffle certain tasks, in which a task normally performed by a physician is transferred to a health care professional with a different, usually lower, level of education and training. Such programs may result in the creation of new types of health care personnel, such as nonmedical sedation 
practitioners, whose function is to assist physicians or to perform specific tasks independently. Selected nurse anesthetists have been trained and certified to accept responsibility for the decisions they make while safely administering propofol/alfentanil sedation under the indirect supervision of an anesthesiologist.

The primary aim of this 30-month retrospective study was to evaluate the clinical outcomes of moderate-to-deep sedation administered by trained nonmedical sedation practitioners using a propofol/opioid technique during GI endoscopy, with a particular emphasis on adverse events related to ventilation and circulation complications.

\section{Patients/materials and methods \\ $\nabla$}

\section{Study population and design}

On September 11, 2013, the UMCU Medical Ethical Committee approved the protocol (No.13-467/C) for this retrospective study, which was registered as reference No.WAG/om/ 13069604. Selected patients were offered sedation because of the earlier failure of light sedation administered by a gastroenterologist and/or because of their medical condition. A total of 597 patients consecutively underwent diagnostic and therapeutic GI endoscopic procedures with moderate-to-deep sedation administered by five trained nonmedical sedation practitioners during a period of 30 months, from September 2013 to July 2014; these patients were included in a database that was retrospectively analyzed. The nonmedical trained sedation practitioners had experience in administering propofol for moderate-todeep sedation in the department of gastroenterology; they also had experience of at least 150 cases in administering moderateto-deep sedation during (interventional) cardiology and pulmonary diagnostic procedures.

The patients were divided into four groups based on increasing procedural complexity: group I, colonoscopy; group II, combined gastroscopy and colonoscopy; group III, esophagogastroduodenoscopy (EGD), defined as upper GI endoscopy and/or a singleballoon endoscopic procedure; and group IV, endoscopic retrograde cholangiopancreatography (ERCP) and endoscopic ultrasound (EUS) ( $\bullet$ Table1).

The medical status of each patient was screened and approved by the trained sedation practitioner. In the case of a patient with American Society of Anesthesiologists (ASA) physical status class III or IV, an anesthesiologist was consulted to determine the most appropriate strategy. The ASA physical status class [11] of each patient was assessed in accordance with the hospital procedural sedation and analgesia screening protocol. The Mallampati clinical scoring system $[12,13]$ was used to predict difficult airway management. Demographic data, duration of the procedures, complications (e.g., hypoventilation, apnea, arterial desaturation,

\begin{tabular}{|ll|}
\hline Table 1 & Endoscopic procedures grouped according to complexity. \\
\hline Group & Procedure \\
\hline I & Colonoscopy \\
\hline II & Combined gastroscopy and colonoscopy \\
\hline III & Esophagogastroduodenoscopy (EGD) \\
\hline & $\begin{array}{l}\text { Percutaneous endoscopic gastrostomy (PEG) } \\
\text { Endoscopic mucosal resection (EMR) }\end{array}$ \\
\hline IV & Zenker's diverticulum \\
\hline & Endoscopic retrograde cholangiopancreatography (ERCP) \\
\hline
\end{tabular}

hypotension, rhythm disturbances, decreased awareness), and data from the recovery period were assessed. Clinical outcomes were analyzed according to the complication list of the Netherlands Society of Anesthaesiology ( Table 2 ).

Following screening, all patients gave informed consent for the sedation. Inclusion criteria for moderate-to-deep sedation were age of 18 years or older and compliance with fasting guidelines before the procedure. Exclusion criteria were allergy to soy, eggs, or peanuts; pregnancy; acute GI bleeding; and mental disability [14].

Trained nonmedical sedation practitioners of the department of anesthesiology screened the health status of the patients, monitored them, provided procedural sedation and analgesia, and recorded their data. These professionals had no concomitant responsibilities at the time. All patients were briefed before the sedation procedure, with an anesthesiologist immediately available by phone for consultation and/or intervention, as documented in a hospital protocol.

\section{Sedation protocol and monitoring}

The depth of sedation was assessed continuously and recorded at least every 5 minutes with the Observer's Assessment of Alertness/Sedation (OAA/S) scale [15], based on a combination of observation of the resting patient and the patient's responses to verbal commands of increasing intensity. The score ranges from 1 (does not respond) to 5 (alert). Before the GI procedure, an intravenous infusion was initiated for fluid administration. The vital signs of all patients were continuously observed and monitored (qube Compact Monitor; Spacelabs Healthcare, Snoqualmie, Washington, USA), and all data were recorded every 5 minutes with AnStat, an anesthesia information management system. Heart activity was monitored with three-lead electrocardiography (ECG) and oxygen saturation with pulse oximetry $\left(\mathrm{SpO}_{2}\right)$. Noninvasive blood pressure (NIBP) measurements were taken at 5-minute intervals, and capnography readings (Smart CapnoLine Plus; Oridion Capnography, Needham, Massachusetts, USA) were continuously recorded. All patients received supplemental oxygen $(2 \mathrm{~L} / \mathrm{min}$ ) by nasal cannula. Procedural sedation and anesthesia started with the intravenous administration via infusion pump (Alaris Medical UK) of $5 \mathrm{mg} / \mathrm{kg} / \mathrm{hrs}$ of propofol (Lipuro $1 \%$ [10 mg/mL]; B. Braun) per hour and $200 \mu \mathrm{g}$ of alfentanil (Janssen-Cilag) as a bolus. Additional intravenous boluses of 10 or 20 $\mathrm{mg}$ of propofol were titrated until the desired level of moderateto-deep sedation (OAA/S sedation score of 4 or 3 ) was achieved. Our goal was to maintain a sedation level between moderate (patient responds to verbal or tactile stimulus) and deep (patient not aroused easily but responds to painful stimuli). Depending on the clinical signs or symptoms of pain, additional intravenous boluses of 100 to $200 \mu \mathrm{g}$ of alfentanil were given.

The primary outcome was to investigate the incidence of adverse events affecting the patient's ventilation and circulation. Unintended disruption of the patient's ventilation was defined as the observation of hypoxemia, aspiration, or laryngospasm. Hypoxemia was defined as oxygen saturation of $92 \%$ or lower for at least 5 minutes and aspiration of gastric contents confirmed by aspiration of fluid in the trachea with a suction catheter. Laryngospasm was defined as stridor or upper airway obstruction associated with a decrease in arterial oxygen saturation, requiring an intervention such as chin lift, jaw thrust, insertion of an oral airway, placement of a laryngeal mask, or even orotracheal intubation. A ventilation complication was defined as mild if an unintended disruption lasted for no longer as 30 seconds and was self-limit- 
Table 2 The national modified complication list of the Netherlands Society of Anaesthesiology.

\begin{tabular}{|c|c|}
\hline Baseline characteristics of the study population & Measurements \\
\hline Patient identification & Baseline first measured systolic NIBP, mean, $\mathrm{mmHg}$ \\
\hline Gastrointestinal endoscopy & Baseline first measured diastolic NIBP, mean, $\mathrm{mmHg}$ \\
\hline Group I & Baseline lowest measured systolic NIBP, mean, $\mathrm{mmHg}$ \\
\hline Colonoscopy & Baseline lowest measured diastolic NIBP, mean, $\mathrm{mmHg}$ \\
\hline Group II & Baseline first measured heart rate, mean, beats/min \\
\hline Combined gastroscopy and colonoscopy & Baseline highest measured heart rate, mean, beats/min \\
\hline Group III & Baseline lowest measured heart rate, mean, beats /min \\
\hline Esophagogastroduodenoscopy & Baseline first measured $\mathrm{SpO}_{2}$, mean, \% \\
\hline Percutaneous endoscopic gastrostomy & Baseline lowest measured $\mathrm{SpO}_{2}$, mean, \% \\
\hline Endoscopic mucosal resection & Baseline last measured $\mathrm{SpO}_{2}$, mean, \% \\
\hline Zenker's diverticulum & Baseline first measured end-tidal $\mathrm{CO}_{2}$, mean, \% \\
\hline Group IV & Last measured end-tidal $\mathrm{CO}_{2}$, mean, $\%$ \\
\hline Endoscopic retrograde cholangiopancreatography & Overall OAA/S score \\
\hline Endoscopic ultrasonography & 5 \\
\hline Baseline characteristics & 4 \\
\hline Age, $y$ & 3 \\
\hline Male & 2 \\
\hline Female & 1 \\
\hline American Society of Anesthesiologists (ASA) classification & Recovery care \\
\hline 1 & First VAS score in recovery room: 0 , no pain; 10 , maximum pain \\
\hline II & First Aldrete score in recovery room \\
\hline III & Events associated with sedation and intervention \\
\hline IV & Systolic hypotension (> $25 \%$ from baseline), $\mathrm{mmHg}$ \\
\hline Mallampati score & Systolic hypertension ( $>25 \%$ from baseline), $\mathrm{mmHg}$ \\
\hline 1 & Bradycardia (>25\% from baseline), beats $/ \mathrm{min}$ \\
\hline II & Tachycardia ( $>25 \%$ from baseline), beats $/ \mathrm{min}$ \\
\hline III & Hypoxemia $\left(\mathrm{SpO}_{2} \leq 92 \%\right)$ \\
\hline IV & Chin lift \\
\hline Monitoring & Aspiration \\
\hline ECG, $\mathrm{SpO}_{2}$, capnography, $\mathrm{O}_{2}$ supply, NIBP & Airway obstruction \\
\hline Procedure time, $\min$ & Oral Guedel airway \\
\hline Intravenous cannula, 20 gauge & Bag valve mask-assisted ventilation \\
\hline Medication during procedure & Oral tracheal intubation \\
\hline Alfentanil dose, $\mathrm{mg}$ & Aborted procedure \\
\hline Propofol dose, $\mathrm{mg}$ & Anesthesiologist advice required \\
\hline Atropine & Complication due to endoscopic procedure \\
\hline Ephedrine & Perforation \\
\hline Lidocaine & Bleeding \\
\hline
\end{tabular}

ECG, electrocardiography; $\mathrm{SpO}_{2}$, oxygen saturation as measured with pulse oximetry; NIBP, noninvasive blood pressure; OAA/S, observer's assessment of alertness/sedation; VAS, Visual Analog Scale.

\begin{tabular}{|c|c|c|c|c|}
\hline ASA class & Group I: Colonoscopy & $\begin{array}{l}\text { Group II: Combined } \\
\text { gastroscopy and } \\
\text { colonoscopy }\end{array}$ & $\begin{array}{l}\text { Group III: EGD, PEG, } \\
\text { EMR, Zenker's } \\
\text { diverticulum }\end{array}$ & $\begin{array}{l}\text { Group IV: } \\
\text { ERCP, EUS }\end{array}$ \\
\hline I & 47 & 3 & $24^{*}$ & 1 \\
\hline II & 97 & 30 & 244 & 54 \\
\hline III & 12 & 3 & 30 & 47 \\
\hline IV & 0 & 0 & 3 & 1 \\
\hline
\end{tabular}

Table 3 Study results according to American Society of Anesthesiologists (ASA) physical status class of patients.

EGD, esophagogastroduodenoscopy; PEG, percutaneous endoscopic gastrostomy; EMR, endoscopic mucosal resection; ERCP, endoscopic retrograde cholangiopancreatography; EUS, endoscopic ultrasonography.

* In one case, the patient's ASA class was not reported.

ing; otherwise, the complication was considered severe. Unintended disruption of the circulation was defined as the registration of hypotension or hypertension. Hypotension was defined as a mean NIBP measurement of $60 \mathrm{mmHg}$ or less for at least $5 \mathrm{~min}$ utes and was treated with vasopressors. Hypertensive periods were defined when the diastolic NIBP was above $110 \mathrm{mmHg}$ and/or the systolic NIBP was above $180 \mathrm{mmHg}$ for at least $5 \mathrm{~min}-$ utes, requiring pharmacologic intervention. A circulation compli- cation was defined as severe if the given drug did not have the desired effect within 30 seconds.

\section{Recovery}

After the procedure, all patients stayed in the recovery room for at least 1 hour and were continuously observed and monitored (ECG, NIBP, and $\mathrm{SpO}_{2}$ ). The modified Aldrete score [16] was recorded on arrival and every 10 minutes in the recovery room. 
Table 4 Age of the patients (Part 1).

\begin{tabular}{|c|c|c|c|c|c|c|c|c|}
\hline \multirow[t]{3}{*}{ Age } & \multicolumn{2}{|c|}{ Group I } & \multicolumn{2}{|c|}{ Group II } & \multicolumn{2}{|c|}{ Group III } & \multicolumn{2}{|c|}{ Group IV } \\
\hline & \multicolumn{2}{|c|}{ Colonoscopy } & \multicolumn{2}{|c|}{$\begin{array}{l}\text { Combined gastroscopy and } \\
\text { colonoscopy }\end{array}$} & \multicolumn{2}{|c|}{$\begin{array}{l}\text { EGD, PEG, EMR, Zenker's } \\
\text { diverticulum }\end{array}$} & \multicolumn{2}{|c|}{ ERCP, EUS } \\
\hline & Male & Female & Male & Female & Male & Female & Male & Female \\
\hline Count & 44 & 112 & 16 & 20 & 160 & 142 & 39 & 64 \\
\hline Mean & 54.8 & 52.9 & 47.6 & 46.6 & 56.4 & 54.8 & 55.4 & 52.8 \\
\hline Minimum & 19 & 20 & 18 & 23 & 19 & 20 & 15 & 15 \\
\hline Maximum & 80 & 89 & 79 & 75 & 86 & 90 & 75 & 88 \\
\hline Standard deviation & 17.93 & 17.09 & 16.78 & 16.87 & 15.5 & 15.29 & 12.23 & 16.88 \\
\hline
\end{tabular}

Table4 Age of the patients (Part 2).

\begin{tabular}{|llllll|}
\hline ANOVA & & & & & \\
\hline Age & Sum of squares & df & Mean square & F & Significant \\
\hline Between groups & $2,574,123$ & 3 & 858,041 & 3.366 & .018 \\
\hline Within groups & $151,151,144$ & 593 & 254,892 & & \\
\hline Total & $153,725,266$ & 596 & & & \\
\hline
\end{tabular}

\begin{tabular}{|c|c|c|c|c|c|c|}
\hline \multicolumn{7}{|c|}{ Bonferroni multiple comparisons } \\
\hline \multicolumn{7}{|l|}{ Age } \\
\hline \multirow[t]{2}{*}{ (I) Group } & \multirow[t]{2}{*}{ (J) Group } & \multirow{2}{*}{$\begin{array}{l}\text { Mean difference } \\
(I-J)\end{array}$} & \multirow[t]{2}{*}{ Standard error } & \multirow[t]{2}{*}{ Significant } & \multicolumn{2}{|l|}{$95 \% \mathrm{Cl}$} \\
\hline & & & & & Lower boundary & Upper boundary \\
\hline \multirow[t]{3}{*}{1} & 2 & 6.419 & 2.952 & .180 & -1.40 & 14.23 \\
\hline & 3 & -2.178 & 1.574 & 1.000 & -6.34 & 1.99 \\
\hline & 4 & -.302 & 2.027 & 1.000 & -5.67 & 5.06 \\
\hline \multirow[t]{3}{*}{2} & 1 & -6.419 & 2.952 & .180 & -14.23 & 1.40 \\
\hline & 3 & $-8.597^{*}$ & 2.815 & .014 & -16.05 & -1.15 \\
\hline & 4 & -6.721 & 3.091 & .180 & -14.90 & 1.46 \\
\hline \multirow[t]{3}{*}{3} & 1 & 2.178 & 1.574 & 1.000 & -1.99 & 6.34 \\
\hline & 2 & $8.597^{*}$ & 2.815 & .014 & 1.15 & 16.05 \\
\hline & 4 & 1.876 & 1.822 & 1.000 & -2.95 & 6.70 \\
\hline \multirow[t]{3}{*}{4} & 1 & .302 & 2.027 & 1.000 & -5.06 & 5.67 \\
\hline & 2 & 6.721 & 3.091 & .180 & -1.46 & 14.90 \\
\hline & 3 & -1.876 & 1.822 & 1.000 & -6.70 & 2.95 \\
\hline
\end{tabular}

EGD, esophagogastroduodenoscopy; PEG, percutaneous endoscopic gastrostomy; EMR, endoscopic mucosal resection; ERCP, endoscopic retrograde cholangiopancreatography; EUS, endoscopic ultrasonography; ANOVA, analysis of variance; $\mathrm{df}$, degrees of freedom; F, F statistic; Cl, confidence interval.

* Significant at $P=0.05$.

This score describes patient motor activity, oxygen saturation, blood pressure, respiratory function, and consciousness. Pain scores were recorded and evaluated every 10 minutes with a Visual Analog Scale (VAS) [17]. The VAS score ranged from 0 (no pain) to 10 (extreme pain). Patients were discharged when full consciousness had been regained, when their vital signs (heart rate, oxygen saturation, NIBP) were within normal limits, when the pain score was 3 or lower [18], and when the modified Aldrete score was 9 or higher and stable for a minimum of 60 minutes.

\section{Statistical analyses}

Statistical analyses were performed with IBM SPSS Statistics, Version 23 (IBM, Armonk, New York, USA). The four endoscopy groups were compared with one-way analysis of variance (ANOVA). This test determines if there is any significant difference be- tween groups by age, duration of the procedure, or gender. The Bonferroni method, as a follow-up test to ANOVA, is used to determine significant differences between groups. For age, $P=0.05$ was considered statistically significant.

\section{Results \\ Safety}

All endoscopic procedures in the 597 patients were carried out efficiently according to plan, and no failures were recorded. An acute intervention by the indirectly supervising anesthesiologist was not required in any of the cases. No mortality and no serious morbidity occurred. The anesthesiologist who conducted the briefing before the sedation procedure decided that moderateto-deep sedation was not indicated in 5 of the 597 patients, in 
Table 5 Mild and severe cases of unintended disruption of ventilation and circulation.

\begin{tabular}{|c|c|c|c|c|c|c|c|c|c|c|c|c|c|c|c|c|c|}
\hline & & \multicolumn{4}{|c|}{ Group I: Colonoscopy } & \multicolumn{4}{|c|}{$\begin{array}{l}\text { Group II: Combined gas- } \\
\text { troscopy and colonoscopy }\end{array}$} & \multicolumn{4}{|c|}{$\begin{array}{l}\text { Group III: EGD, PEG, EMR, } \\
\text { Zenker's diverticulum }\end{array}$} & \multicolumn{4}{|c|}{ Group IV: ERCP, EUS } \\
\hline & & $\begin{array}{l}\text { ASA } \\
\text { I }\end{array}$ & $\begin{array}{l}\text { ASA } \\
\text { II }\end{array}$ & $\begin{array}{l}\text { ASA } \\
\text { III }\end{array}$ & $\begin{array}{l}\text { ASA } \\
\text { IV }\end{array}$ & $\begin{array}{l}\text { ASA } \\
\text { I }\end{array}$ & $\begin{array}{l}\text { ASA } \\
\text { II }\end{array}$ & $\begin{array}{l}\text { ASA } \\
\text { III }\end{array}$ & $\begin{array}{l}\text { ASA } \\
\text { IV }\end{array}$ & $\begin{array}{l}\text { ASA } \\
\text { I }\end{array}$ & $\begin{array}{l}\text { ASA } \\
\text { II }\end{array}$ & $\begin{array}{l}\text { ASA } \\
\text { III }\end{array}$ & $\begin{array}{l}\text { ASA } \\
\text { IV }\end{array}$ & ASA & $\begin{array}{l}\text { ASA } \\
\text { II }\end{array}$ & $\begin{array}{l}\text { ASA } \\
\text { III }\end{array}$ & $\begin{array}{l}\text { ASA } \\
\text { IV }\end{array}$ \\
\hline \multicolumn{18}{|c|}{ Unintended disruption of ventilation } \\
\hline Aspiration & $\mathrm{n}=0$ & & & & & & & & & & & & & & & & \\
\hline \multicolumn{18}{|l|}{ Laryngospasm } \\
\hline Intubation & $\mathrm{n}=2(0.3 \%)$ & & & & & & & & & $1^{*}$ & & $1^{*}$ & & & & & \\
\hline Mask ventilation & $\mathrm{n}=7(1.1 \%)$ & & & 1 & & & 1 & & & 1 & 3 & 1 & & & & & \\
\hline Chin lift & $\mathrm{n}=17(2.8 \%)$ & & & & & & & & & 1 & 15 & 1 & & & & & \\
\hline Guedell & $\mathrm{n}=4(0.7 \%)$ & & & & & & & & & 1 & 2 & 1 & & & & & \\
\hline \multicolumn{18}{|l|}{ Hypoxemia } \\
\hline $\mathrm{SpO}_{2} 92 \%$ & $\mathrm{n}=12(2 \%)$ & & 1 & & & & & & & & 11 & & & & & & \\
\hline $\mathrm{SpO}_{2} 91 \%$ & $\mathrm{n}=8(1.3 \%)$ & 1 & 2 & & & & 1 & & & & 1 & 1 & & & 2 & & \\
\hline $\mathrm{SpO}_{2} 85 \%-90 \%$ & $\mathrm{n}=8(1.3 \%)$ & & & 1 & & & 1 & & & & 6 & & & & & & \\
\hline $\mathrm{SpO}_{2}<85 \%$ & $\mathrm{n}=17(2.8 \%)$ & & & & & & & & & $1^{*}$ & 15 & $1^{*}$ & & & & & \\
\hline \multicolumn{18}{|c|}{ Unintended disruption of circulation } \\
\hline \multicolumn{18}{|l|}{ NIBP } \\
\hline Mean $<60 \mathrm{mmHg}$ & $\mathrm{n}=7(1.2 \%)$ & & 1 & 3 & & & & & & & 1 & & & & 2 & & \\
\hline $\begin{array}{l}\text { Diastolic }>110 \\
\mathrm{mmHg} \text {, systolic } \\
>80 \mathrm{mmHg}\end{array}$ & $\mathrm{n}=7(1.2 \%)$ & 1 & & & & 1 & & & & & 3 & 2 & & & & & \\
\hline
\end{tabular}

EGD, esophagogastroduodenoscopy; PEG, percutaneous endoscopic gastrostomy; EMR, endoscopic mucosal resection; ERCP, endoscopic retrograde cholangiopancreatography; EUS, endoscopic ultrasonography; ASA, American Society of Anesthesiologists; $\mathrm{SpO}_{2}$, oxygen saturation as measured by pulse oximetry; NIBP, noninvasive blood pressure. * Severe complication.

whom sedation was converted to general anesthesia by an anesthesia team.

\section{Demographic data}

The distribution of the ASA physical status scores was as follows: ASA I, 75 patients (13\%); ASA II, 426 patients (71\%); ASA III, 92 patients (15\%); and ASA IV, 4 patients $(<1 \%)$. In one case, the ASA classification score was not reported $(\bullet$ Table 3$)$.

\section{Patient age and procedure duration}

The mean age of the patients between the four groups varies significantly $(P=0.018, \otimes$ Table 4). Groups II and III appear to differ significantly from each other $(P=0.014)$, whereas there is no significant proof of a difference for the other groups $(P>0.1)$. A linear regression with $P<0.001$ indicating significance shows a positive correlation between patient age and procedure duration.

Incidence of hypoxemia, hypotension, and hypertension Mild complications ( $\triangle$ Table 5) were recorded in 85 of the 597 patients, and severe complications in 4 of the 597 patients. Hypoxemia was a severe complication (desaturation lasting longer than 5 minutes and requiring intubation of the trachea) in $2 \mathrm{pa}-$ tients ( $0.3 \%)$ and a mild complication in 43 patients (7.2\%). Laryngospasm, which was treated with chin lift, mask ventilation, or insertion of an oral airway, was characterized as a mild complication in 28 patients (4.7\%) and as a severe complication in 2 patients $(0.3 \%)$.

No signs or symptoms suggestive of aspiration were reported.

Mild blood pressure-related complications were as follows: hypotension in 7 patients $(1.2 \%)$, which was treated with vasopressors; hypertension in 7 patients, which was managed by increasing the dose of the sedative and/or opioid. All procedures were successfully completed.

Moderate-to-deep sedation provided by trained sedation practitioners resulted in efficient endoscopic GI procedures in all cases.
Among the 597 patients, 11 with a sedation score of OAA/S 3 or 4 spontaneously reported awareness as a sign of an insufficient level of sedation during the procedure.

\section{Recovery and discharge}

Upon arrival in the recovery room, 177 patients (29.6\%) had the maximum Aldrete score of 10 .There were 168 patients ( $28.1 \%$ ) with a score of 9,134 patients $(22.4 \%)$ with a score of 8 , and 74 patients $(12.3 \%)$ with a score of 7 . Fewer than $10 \%$ of all patients had an Aldrete score between 6 and 2.Furthermore, upon arrival in the recovery room, 246 patients $(41 \%)$ had a minimum VAS score of 0,114 patients (19\%) had a score of 1 , and 69 patients (12\%) had a score of 2 ( $\bullet$ Table 6 ).

\section{Discussion \\ $\nabla$}

As a result of the exponential increase in the number of diagnostic and therapeutic GI endoscopic procedures being performed in the last few decades, there is a great demand for sedation services, which in most countries cannot be met by anesthesiologists because of a shortage of these specialists. Meanwhile, endoscopists have been looking for alternative solutions to increase the efficiency of endoscopic procedures and to improve their working conditions with the use of sedative drugs, often in uncontrolled or moderately controlled conditions.

Quality and safety usually have not been primary considerations in the development of these solutions, which rapidly became popular because of their ease of application. A number of articles have discussed the use of propofol by clinicians [19, 20], mostly gastroenterologists, who have no training in anesthesia or resuscitation. In contrast, anesthesiologists, in particular in the United States, abide by the official Food and Drug Administration (FDA) standpoint that propofol should be administered only by persons trained in general anesthesia [21]. However, an increasing num- 


\begin{tabular}{|c|c|c|c|}
\hline \multicolumn{2}{|l|}{ Aldrete scoring system } & \multicolumn{2}{|c|}{ First Aldrete score after each procedure } \\
\hline Respiration & Score & Score & Patient data \\
\hline Able to take deep breath and cough & 2 & 10 & $n=177(30 \%)$ \\
\hline Dyspnea/shallow breathing & 1 & 9 & $\mathrm{n}=168(28 \%)$ \\
\hline Apnea & 0 & 8 & $n=134(22 \%)$ \\
\hline Activity & & 7 & $\mathrm{n}=74(12 \%)$ \\
\hline Able to move 4 extremities & 2 & 6 & $n=23(4 \%)$ \\
\hline Able to move 2 extremities & 1 & 5 & $n=11(2 \%)$ \\
\hline Able to move 0 extremities & 0 & 4 & $\mathrm{n}=6(1 \%)$ \\
\hline Circulation & & 3 & $\mathrm{n}=2(<1 \%)$ \\
\hline NIBP $20 \mathrm{mmHg}$ before sedation & 2 & 2 & $\mathrm{n}=2(<1 \%)$ \\
\hline NIBP $20-50 \mathrm{mmHg}$ before sedation & 1 & 1 & $\mathrm{n}=0$ \\
\hline Consciousness & & \multicolumn{2}{|c|}{ VAS score before release/recovery } \\
\hline Fully awake & 2 & Score & Patient data \\
\hline Arousable on calling & 1 & 0 & $n=246(41 \%)$ \\
\hline Not responding & 0 & 1 & $\mathrm{n}=114(19 \%)$ \\
\hline $\mathrm{SpO}_{2}$ on room air & & 2 & $\mathrm{n}=69(12 \%)$ \\
\hline $100 \%-98 \%$ & & 3 & $\mathrm{n}=18(3 \%)$ \\
\hline $97 \%-95 \%$ & & 4 & $\mathrm{n}=28(5 \%)$ \\
\hline$<95 \%$ & & 5 & $n=24(4 \%)$ \\
\hline \multirow[t]{5}{*}{ Total score } & & 6 & $n=21(4 \%)$ \\
\hline & & 7 & $\mathrm{n}=8(1 \%)$ \\
\hline & & 8 & $\mathrm{n}=8(1 \%)$ \\
\hline & & 9 & $n=2(<1 \%)$ \\
\hline & & 10 & $\mathrm{n}=1(<1 \%)$ \\
\hline
\end{tabular}

Table 6 Modified Aldrete score and visual analog scale (VAS) score.

NIBP, noninvasive blood pressure; VAS, Visual Analog Scale; $\mathrm{SpO}_{2}$, oxygen saturation as measured by pulse oximetry.

ber of published reports [22,23] show a very good safety record for sedation administered by clinicians other than anesthesiologists, even nonmedical practitioners. These findings are in contrast with data from a study published in 2002 [24]. We hereby report on our first experiences with 597 consecutive patients undergoing GI endoscopic procedures with moderate-to-deep sedation administered by trained anesthesia nurses. The patients eligible for moderate-to-deep sedation during endoscopy were clustered and treated in a full-day program.

In our retrospective study, all GI endoscopic procedures (100\%) were completed successfully with the patients under moderateto-deep sedation administered by nonmedical sedation practitioners. None of our procedures, even technically difficult and time-consuming ones such as ERCP and EUS, had to be interrupted because of insufficient cooperation or unrest of the patient or because of severe complications.

In our 597 procedures, mild hypoxemia occurred in $7.2 \%$ of the cases and mild laryngospasm in $4.7 \%$ of the cases, and both complications could be managed easily by the trained sedation practitioners. Endotracheal intubation was required in only 2 patients $(0.3 \%)$, in one because of increasing stridor and in the other because of blood loss from the upper GI tract as a complication of the endoscopic procedure. In these two cases, the assistance of an anesthesiologist was required.

Hypotension was observed in $1.2 \%$ of the patients and hypertension in another $1.2 \%$, both considered mild cardiovascular complications.

Berzin [25] reported 140 events in 109 patients (20.6\%) during 528 ERCP procedures in which sedation was administered by anesthesiologists. The events were often a combination of hypotension (38), arrhythmia (20), oxygen desaturation to less than $85 \%$ (66), and unplanned intubation (16) that could occur during one procedure. Our rate of mild complications was lower. This under- scores the need for good patient selection, well-trained sedation practitioners, careful monitoring, and the practitioner's undivided attention for each patient. Wehrmann and Riphaus [26], in a 6-year study of 9547 patients, reported a total of 135 adverse events (1.4\%) during endoscopic procedures conducted with the patients under propofol sedation. In their study, the events were mainly assisted ventilation $(0.4 \%)$ and endotracheal intubation (0.09\%), and 4 patients died (mortality rate of $0.04 \%$ ). Agostoni et al. [27], in an 8-year study, reported adverse events in $4.5 \%$ of 17,999 patients undergoing target-controlled propofol sedation for GI endoscopic procedures. There were 6 complications (arterial hypotension, desaturation, bradycardia, arterial hypertension, arrhythmia, and aspiration) occurring in more than $0.1 \%$ of the patients, and 3 patients died $(0.017 \%)$. In both studies, the overall incidence of adverse events was lower than that in our study, although differences in definitions may play a role.

We agree with the findings and the conclusion of Wehrmann and Riphaus that interventional endoscopy under propofol sedation is not risk-free, and that monitoring of the patient's vital parameters during GI interventions is necessary.

Because several studies $[28,29]$ have shown a variety of moderate-to-deep sedation-related complications in GI endoscopy, it is mandatory, in our opinion, that moderate-to-deep sedation be administered by a well-trained practitioner who is competent in handling acute respiratory and circulatory events [30]. This is especially true for some subcategories of patients. We observed a trend toward a greater incidence of unwanted side effects in a specific group of patients - namely, those undergoing EGD, defined as upper GI endoscopy and/or a single-balloon endoscopy procedure; this was particularly noted in ASA class I and II patients more than in ASA class III patients.

It has also been shown by other investigators $[31,32]$ that propofol-based sedation can be administered safely to patients by well- 
trained nonmedical sedation practitioners whose attention is solely devoted to administering sedation and who are not participating in the endoscopic procedure itself [33]. Propofol-based sedation is characterized by a narrow therapeutic window $[34,35]$. In our study, the nonmedical sedation practitioners had no responsibility other than to administer sedation and to observe and monitor the patients.

We provided sedation to our patients with a continuous infusion of propofol $(5 \mathrm{mg} / \mathrm{kg} / \mathrm{h})$ followed by intermittent titrated doses of alfentanil $(100-200 \mu \mathrm{g})$. This strategy in relation to the severity of the stress response has been shown to contribute to cardiovascular stability during sedation.

Capnography was used to monitor respiratory activity [36], as an early warning system for hypoventilation and impending hypoxemia. In our experience, this mode of monitoring, which now is easily applied as a result of newly developed sampling techniques in combination with disposable pulse oximeter sensors, contributes significantly to the early detection of impending hypoxemia and may improve patient safety during procedural sedation and analgesia in GI endoscopy. Our experience is in contrast to the study of van Loon et al. [37], in which $100 \mathrm{mg}$ of propofol was given as an initial dose to 427 patients and no alveolar plateau appeared in the capnogram before hypoxemia occurred. Therefore, use of the OAA/S scale, which assesses the patient's level of alertness, is also mandatory during moderate-to-deep sedation. These findings are in agreement with those of Lera dos Santos et al. [38], who evaluated the use of OAA/S scores determined every 2 minutes and found an overall rate of deep sedation of $18 \%$ as a result of using the observational alertness tool.

\section{Conclusion}

\section{$\nabla$}

For a risky medical procedure such as moderate-to-deep sedation during GI endoscopic procedures, strict safety conditions have to be met. Improvement in the quality of sedation care achieved by coordinating practices will contribute to quality, safety, and patient comfort. Our study shows that in the Netherlands, well-trained nonmedical sedation practitioners can be entrusted with the responsibility of safely administering propofol/ opioid-based moderate-to-deep sedation in selected patients undergoing GI endoscopic procedures.

Serious complications with propofol-based sedation, especially respiratory and cardiovascular adverse events, may occur but were rare in our safety setting. These complications need to be recognized rapidly by pro-active monitoring and could be appropriately managed by skilled practitioners. Our data may be useful for the future planning of new clinical strategies in this setting in Europe.

\section{Competing interests: None}

\section{References}

1 Hazeldine S, Fritschi L, Forbes G. Predicting patient tolerance of endoscopy with conscious sedation. Scand J Gastroenterol 2010; 45: 1248 1254

2 Zakko SF, Seifert HA, Gross JB. A comparison of midazolam and diazepam for conscious sedation during colonoscopy in a prospective double-blind study. Gastrointest Endosc 1999; 49: 684-689

3 Qadeer MA, Lopez AR, Dumot JA et al. Hypoxemia during moderate sedation for gastrointestinal endoscopy: causes and associations. Digestion 2011; 84: 37-45

4 Girdler NM, Fairbrother KJ, Lyne JP et al. A randomised crossover trial of post-operative cognitive and psychomotor recovery from benzodiaze- pine sedation: effects of reversal with flumazenil over a prolonged recovery period. Br Dent J 2002; 192: 335-339

5 Stanley $\mathrm{TH}$. The history and development of the fentanyl series. J Pain Symptom Manage 1992; 7 Suppl 3: 3-S7

6 Patel S, Vargo J, Khandwala F et al. Deep sedation occurs frequently during elective endoscopy with meperidine and midazolam. Am J Gastroenterol 2005; 100: 2689-2695

7 Heuss LT, Sughandaa SP, Degen LP. Endoscopy teams' judgment of discomfort among patients undergoing colonoscopy - how bad was it really? Swiss Med Wkly 2012; 142: w13726

8 Cohen LB, Wecsler JS, Gaetano JN et al. Endoscopic sedation in the United States: results from a nationwide survey. Am J Gastroenterol 2006; 101: $967-974$

9 Jung $M$, Hofmann $C$, Kiesslich $R$ et al. Improved sedation in diagnostic and therapeutic ERCP: propofol is an alternative to midazolam. Endoscopy 2000; 32: 233-238

10 Knape JTA, van Everdingen JJE. Consensus sedatie en analgesie door artsen-niet-anesthesiologen. Ned Tijdschr Geneeskde 1999; 143: 1098 1102

11 Mak PH, Campbell RC, Irwin MG. American Society of Anesthesiologists. The ASA Physical Status Classification: inter-observer consistency. American Society of Anesthesiologists. Anaesth Intensive Care 2002; 30: 633-640

12 Mallampati SR. Clinical sign to predict difficult tracheal intubation (hypothesis). Can Anaesth Soc J 1983; 30: 316-317

13 Mallampati SR, Gatt SP, Gugino LD et al. A clinical sign to predict difficult tracheal intubation: a prospective study. Can Anaesth Soc J 1985; 32: $429-434$

14 Dripps $R D$. New classification of physical status. Anesthesiology 1963; 24: 111

15 Chernik DA, Gillings D, Laine $H$ et al. Validity and reliability of the observer's assessment of alertness/sedation scale: study with intravenous midazolam. J Clin Psychopharmacol 1994; 10: 244-251

16 Aldrete JA. The post-anesthesia recovery score revisited. J Clin Anesth 1995; 7: 89-91

17 Frieling T, Heise J, Kreysel C et al. Sedation-associated complications in endoscopy prospective multicentre survey of 191142 patients. Z Gastroenterol 2013; 51: $568-572$

18 McCaffery M, Pasero C. Using the 0-to-10 pain rating scale. Am J Nurs 2001: 101; 81-82

$19 \mathrm{Kim} \mathrm{SG}$. The elderly also deserves to undergo therapeutic endoscopy safely under sedation with propofol by gastroenterologists. Gut Liver 2015; 9: 1-2

20 García-Suárez C, López-Rosés L, Olivencia P et al. Sedation with propofol controlled by endoscopists during percutaneous endoscopic gastrostomy. Rev Esp Enferm Dig 2010; 102: 249-256

21 Garewal D, Waikar P. Propofol sedation for ERCP procedures: a dilemna? Observations from an anesthesia perspective Diagn Ther Endosc 2012; 2012: 639190

22 Frieling T, Heise J, Kreysel C et al. Sedation-associated complications in endoscopy - prospective multicentre survey of 191142 patients. Z Gastroenterol 2013; 51: 568-572

23 Külling $D$, Orlandi $M$, Inauen $W$. Propofol sedation during endoscopic procedures: how much staff and monitoring are necessary? Gastrointest Endosc 2007; 66: $443-449$

24 Eisen M, Baron T, Dominitz J et al. Complications of upper GI endoscopy. Gastrointest Endosc 2002; 55: 784 - 793

25 Berzin TM, Sanaka S, Barnett SR et al. A prospective assessment of sedation-related adverse events and patient and endoscopist satisfaction in ERCP with anesthesiologist-administered sedation. Gastrointest Endosc 2011; 73: 710-717

26 Wehrmann T, Riphaus A. Sedation with propofol for interventional endoscopic procedures: a risk factor analysis. Scand J Gastroenterol 2008; 43: $368-374$

27 Agostoni M, Fanti L, Gemma M et al. Adverse events during monitored anesthesia care for GI endoscopy: an 8-year experience. Gastrointest Endosc 2011; 74: 266-275

28 Amornyotin S, Chalayonnawin W, Kongphlay S. Deep sedation for endoscopic retrograde cholangiopancreatography: a comparison between clinical assessment and Narcotrend(TM) monitoring. Med Devices (Auckl) 2011; 4: 43-49

29 Nayar DS, Guthrie WG, Goodman A et al. Comparison of propofol deep sedation versus moderate sedation during endosonography. Dig Dis Sci 2010; 55: 2537-2544 
30 Amornyotin S, Chalayonnavin W, Kongphlay S. Propofol-based sedation does not increase rate of complication during percutaneous endoscopic gastrostomy procedure. Gastroenterol Res Pract 2011; 2011: 134819

31 American Society of Anesthesiologists Task Force on Sedation and Analgesia by Non-Anesthesiologists. Practice guidelines for sedation and analgesia by non-anesthesiologists. Anesthesiology 2002; 96: 1004 1017

32 Yusof F, Raymond G, Sahai AV. Endoscopist administered propofol for upper-GI EUS is safe and effective: a prospective study in 500 patients. Gastrointest Endosc 2004; 60: 356 - 360

33 Deenadayalu VP, Eid EF, GoffJS et al. Non-anesthesiologist administered propofol sedation for endoscopic procedures: a worldwide safety review. Gastrointest Endosc 2008; 67: AB107
34 Faigel DO, Baron TH, Goldstein JL et al. Guidelines for the use of deep sedation and anesthesia for GI endoscopy. Gastrointest Endosc 2002; 5 : $613-617$

35 Nelson DB, Barkun AN, Block KP et al. Propofol use during gastrointestinal endoscopy. Gastrointest Endosc 2001; 7: 876-879

36 Qadeer MA, Vargo JJ, Dumot JA et al. Capnographic monitoring of respiratory activity improves safety of sedation for endoscopic cholangiopancreatography and ultrasonography. Gastroenterology 2009; 136: $1568-1576$

37 van Loon K, van Rheineck Leyssius AAT, van Zaane B et al. Capnography during deep sedation with propofol by non-anesthesiologists: a randomized controlled trial. Anesth Analg 2014; 119: 49-55

38 Lera dos Santos ME, Maluf-Filho F, Chaves DM et al. Deep sedation during gastrointestinal endoscopy: propofol-fentanyl and midazolamfentanyl regimens. World J Gastroenterol 2013; 19: 3439-344 